\title{
Spray on skin for diabetic foot ulcers: an open label randomised controlled trial
}

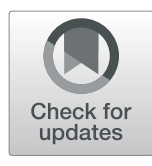

Laurens Manning $^{1 *}$ (D), Emma J. Hamilton², Edward Raby ${ }^{1}$, Paul E. Norman ${ }^{3,4}$, Wendy Davis ${ }^{3}$, Fiona Wood $^{5}$, Keryln Carville ${ }^{6}$, Mendel Baba ${ }^{7}$, Jonathan Hiew ${ }^{7}$, Erica Ryan ${ }^{7}$, Ivana Ferreira ${ }^{7}$, Paul Gittings ${ }^{8}$ and Jens C. Ritter ${ }^{9}$

\begin{abstract}
Background: One Australian loses a limb every $3 \mathrm{~h}$ as a result of infected diabetic foot ulcers (DFU). This common condition accounts for substantial morbidity and mortality for affected individuals and heavy economic costs for the health sector and the community. There is an urgent need to test interventions that improve wound healing time, prevent amputations and recurrent ulceration in patients presenting with DFU whilst improving quality of life and reducing health care costs.
\end{abstract}

Methods: One hundred and fifty eligible participants will be randomised to receive an autologous skin cell suspension, also termed 'spray-on' skin (ReCell ${ }^{\otimes}$ ) or standard care interventions for their DFU. The primary outcome is complete wound healing at 6 months, but participants will be followed up for a total of 12 months to enable secondary outcomes including total overall costs, ulcer free days at 12 months and quality of life to be assessed.

Discussion: Outpatient costs for dressings, home nursing visits and outpatient appointments are key cost drivers for DFU. If spray-on skin is effective, large cost savings to WA Health will be realised immediately through a shortened time to healing, and through a higher proportion of patients achieving complete healing. Shortened healing times may enable participants to return to work earlier. Any economic benefits are likely to be amplified across Australia and other similar demographic settings where aging populations with increased diabetes rates are considered major future challenges.

Trial registration: Australian New Zealand Clinical Trials Registry ACTRN12618000511235. Registered on 9 April 2018.

Keywords: Diabetic foot ulcer, Wound healing, ReCell ${ }^{\circledast}$, Autologous skin cell harvest

\section{Background}

One Australian loses a limb every $3 \mathrm{~h}$ as a direct consequence of diabetes related foot disease, usually due to an infected diabetic foot ulcer (DFU) [1,2]. That amounts to around 8000 lower extremity amputations (LEA) undertaken in Australia each year [3, 4]. In Western Australia (WA) alone, more than 200 major (above the ankle) LEA are performed annually and recurrent minor (below the ankle) amputations in patients with Type 2 diabetes (T2DM) are increasing by about $3.5 \%$ per annum [5]. There has been a $30 \%$ increase in diabetes-related amputations, largely related to increasing diabetes prevalence [6] in Australia over the past decade and 8\% of diabetesrelated deaths are attributable to foot complications $[1,2$,

\footnotetext{
* Correspondence: laurens.manning@uwa.edu.au

'Department of Infectious Diseases, Fiona Stanley Hospital, 11 Robin Warren Drive, Murdoch, WA 6150, Australia

Full list of author information is available at the end of the article
}

7]. These poor outcomes have persisted despite increasing awareness of the medical, economic and social burden of diabetic foot complications [8]. The economic costs and mortality rates exceed that of many common cancers; the 5 -year mortality rate of patients with diabetic foot infections is $~ 50 \%$ [9-12]. The estimated economic burden in Australia may exceed $\$ 1.5$ billion, with DFU accounting for $33 \%$ of diabetes related costs [13]. Poor outcomes associated with DFU are disproportionately high in the Australian Indigenous population [14]. A recently published systematic review has found that Aboriginal and Torres Strait Islander Australians are 3-6 times more likely to experience a diabetes related foot complication than nonAboriginal Australians [15]. Therefore there is an urgent need to test interventions to improve healing time, reduce recurrent ulceration and the incidence of LEA in patients with DFU whilst optimising function and quality of life.

(C) The Author(s). 2019 Open Access This article is distributed under the terms of the Creative Commons Attribution 4.0 International License (http://creativecommons.org/licenses/by/4.0/), which permits unrestricted use, distribution, and reproduction in any medium, provided you give appropriate credit to the original author(s) and the source, provide a link to the Creative Commons license, and indicate if changes were made. The Creative Commons Public Domain Dedication waiver (http://creativecommons.org/publicdomain/zero/1.0/) applies to the data made available in this article, unless otherwise stated. 
In addition to in-patient services required when the DFU becomes limb or life threatening, most of the morbidity and economic burden of DFU is carried by public sector outpatient services. Wound healing time is a key cost driver and influences the overall cost-benefit analysis for any DFU intervention [16]. It is estimated that the mean healing time for a DFU managed without amputation is 6 months, rising to 12 months if an amputation is required $[17,18]$. The outpatient component of management accounts for $71 \%$ of the total costs associated with DFU and suggests that reductions in the time to healing are likely to have major benefits for direct costs, particularly related to home nursing visits, dressings and outpatient appointments.

\section{Rationale}

We hypothesise that the use of 'spray-on' autologous skin grafting (ReCell ${ }^{\circ}$; Avita Medical) in DFU will decrease healing time and thereby reduce overall cost of treatment. Autologous 'spray on' skin aids epithelial regeneration and has been used successfully in the treatment of scars and burns and other ulcers [19], particularly when traditional split skin grafting is not feasible. Although it has shown some early promise in a small case series of $4 \mathrm{ft}$ ulcers [20] and for other chronic ulcers [19], no randomised trial of this product has been completed or is currently planned. The aim of this study is to assess the potential benefit of spray-on skin as a superior, and cost-effective, management strategy for DFU.

\section{Hypotheses}

1. Spray-on autologous skin grafting improves wound healing time in patients with DFU compared with standard care

2. Spray-on autologous skin grafting is cost-effective when compared with standard care

\section{Study design}

This study is a prospective, randomised, open label trial powered for superiority. Local and international data informed sample size calculations indicating that, at present, $45 \%$ of patients with DFU will achieve complete healing at 6 months $[17,18,21]$. We estimate that 136 (with continuity correction) patients are required to have $80 \%$ chance of detecting, at the $5 \%$ level of significance, an increase in the primary outcome measure from $45 \%$ in the control group to $70 \%$ in the spray-on skin group [19]. To account for drop-outs, we will aim to recruit 150 participants in total.

\section{Study population}

The study population will be screened from patients attending the Fiona Stanley (FSH) or Royal Perth
Hospital (RPH) inpatient or outpatient multidisciplinary foot units during the recruitment period. The primary ulcer requiring treatment will be considered the index ulcer for enrolment. The site of the index ulcer will be defined at enrolment and categorised as either being fore-, mid- or hindfoot. During the lead-in phase, the wound bed preparation will be standardised prior to randomisation. Recruitment will occur over an 18-month period and participants followed for 12 months.

\section{Randomisation}

Randomisation will take place 2 weeks (+/ -6 days) from the last significant debridement or minor amputation to allow the surgical wound site to demonstrate early healing. Randomisation into treatment $\left(\operatorname{ReCell}^{\circ}\right)$ or control (standard care) is performed by randomisation program (REDCap) and researchers are blinded to the randomisation algorithm that will include variable block sizes that are randomly 2,4 or 8 .

\section{Inclusion criteria}

i) Age $\geq 18$ years

ii) Diabetes (type 1 or 2 ) defined according to international consensus guidelines

iii) Admission to FSH or RPH, or visit to outpatients departments with a DFU requiring local debridement or minor amputation

iv) Ulcer area $>6 \mathrm{~cm}^{2}$

v) The ulcer location, contour, shape and wound base is deemed to be suitable for administration of spray on skin

vi) No further debridement or amputation is anticipated

vii) Wound bed is adequately vascularised as determined by the presence of at least one palpable pulse in the affected foot, or at least single vessel run off identified by arterial Doppler ultrasonography, MRI, CT or conventional angiography (including following revascularisation procedures)

viii)Competent and willing to provide informed consent

ix) Able to be followed up by ambulatory care services (Silver Chain) for community nursing

\section{Exclusion criteria}

i) Non-diabetic ulcer

ii) Wounds deemed unsuitable on the basis of contour, location, vascularity or other factors

iii) Limb threatening ischaemia or sepsis requiring early major amputation

iv) Not competent to provide informed consent 
v) Unlikely to be accessible for follow-up visit over the next 12 months

\section{Primary outcome}

The primary outcome for the trial will be a dichotomous outcome of complete healing of the index ulcer at 6 months as defined by full epithelialisation, after debridement of callus, lasting for at least 2 weeks. Primary outcome arbitration at the interim analysis and at the final analysis will be performed using the database, wound dimension and clinical images assessed by two independent senior clinicians (not investigators) blinded to the intervention. Discordant outcome assessments will be resolved by consensus.

\section{Secondary outcomes}

Secondary outcomes include: i) index ulcer free days at 12 months, ii) time to full epithelialisation of the index ulcer, iii) trajectory of wound healing of the index ulcer (defined as volume and measured using Silhouette $\left.{ }^{\mathrm{Tm}}\right)$, iv) major adverse events, v) any minor or major lower limb amputation, vi) all-cause mortality, vii) re-ulceration of the index ulcer, viii) the development of any new ulcers ix) total costs of inpatient and outpatient costs (see economic analysis, below), $\mathrm{x})$ readmission to hospital and xi) health-related quality of life (as measured by EQ-5D-5 L) [22].

The definitions for secondary outcomes are consistent with international guidelines [23]. Re-ulceration is defined as healing of index ulcer followed by subsequent ulceration with loss of epithelialisation at the same location. A minor amputation is an amputation below the ankle including toe, metatarsal-phalangeal and midfoot amputations. A major amputation is an amputation above the ankle including below knee and above knee amputations. New onset infection includes infections that have commenced during the preceding 2 weeks, including existing ulcers that have never previously been infected.

\section{Methods}

\section{Pre-intervention - wound bed preparation}

To optimise the quality of the wound bed prior to administration of autologous spray-on skin, wound bed preparation will be standardised; all patients identified for inclusion into the trial will be receive the same preintervention protocol before randomisation. For wounds $>1 \mathrm{~cm}$ deep, negative pressure dressings will be applied with a Prontosan ${ }^{\bullet}$ [B. Braun] soak at each change. For wounds $<1 \mathrm{~cm}$ deep, IntraSite conformable [Smith \& Nephew] (with Prontosan ${ }^{\circ}$ soak as above) will be used.

On the day of randomisation, the index wound will be further cleansed via ultrasonic debridement to remove as much biofilm and devitalised tissue as possible. This will allow for uniformity in wound base appearance prior to randomisation. After ultrasonic debridement, wounds will be swabbed and the swabs stored for future microbiome analysis.

\section{Intervention - spray on skin}

Through a series of validated steps, ReCell $^{\circ}$ [Avita Medical] enables disaggregation of cells from a patient's skin and the preparation of a suspension of these cells that can be sprayed or dropped directly onto the prepared wound bed.

The skin from which the suspension is prepared comes from a small split skin graft (SSG) collected from the patients' upper thigh. An electric dermatome set at $2 \mathrm{~mm}$ depth or a scalpel blade (size 10) placed at a shallow angle will be used to harvest the skin. In comparison with traditional SSG, the size of the sample for the spray on skin preparation is much smaller and will be approximately $2 \mathrm{~cm}^{2}$. The harvest site area will be recorded to control for the dose of skin cells applied as a possible co-variate in statistical analyses.

A small amount of the harvested skin preparation will be reapplied to the harvest site to enable more rapid healing. The harvest site will be dressed with Surfasoft ${ }^{\circ}$ [Tauren] and Mepilex ${ }^{\circ}$ Border [Mölnlycke].

The process of disaggregating the cells is performed with $\mathrm{ReCell}^{\circ}$ [Avita Medical] as previously described in [20] and relies on the enzyme trypsin to allow the epidermis to be separated from the dermis. The cells at the epidermal-dermal junction can then be scraped off using a scalpel and are collected and filtered before being dropped or sprayed onto the wound site. Immediately after autologous skin application the index wound will be dressed with Surfasoft ${ }^{\circ}$ dressing for a minimum of 5 days. Secondary absorbent dressings may be changed as required without disturbing the Surfasoft ${ }^{\circ}$ layer during this time to allow adequate cell adherence.

Patients randomised into the control arm will continue with standard wound care procedures as per normal day to day proceedings of the hospital Outpatients clinic following ultrasonic debridement.

\section{Follow up measurements}

Patients will be followed up at predefined time points. This will coincide with routine outpatient visits ordinarily scheduled at 4, 10, 18, 26, 39 and 52 weeks from randomisation with phone consultations every fortnight to determine total 'ulcer-free' days following healing. At each scheduled visit, the wound will be assessed in terms of location (exact; forefoot vs. hind- and mid-foot), depth, dimensions, wound volume and wound area (as measured by Silhouette ${ }^{\mathrm{mm}}$ [Aranz Medical]), wound quality (patchy vs. confluent, \% epithelialisation), probe to bone test and photography. Both feet will be assessed for new wounds. Blood tests will be (lipids, C-reactive protein, full blood examination and renal and liver function tests) will be 
performed as clinically indicated, but an $\mathrm{HbA}_{1 \mathrm{c}}$ will be measured at 3, 6 and 12 months to determine the change from recruitment. All patients will receive standard care related to off-loading, ongoing diabetes management and infection management as per the usual management protocol of the multi-disciplinary foot ulcer teams. In addition to baseline, an EQ-5D-5 L [22] will be performed at 26 and 52 week visits to ascertain change in healthrelated quality of life [24]. The primary outcome will be assessed at the 26-week visit.

\section{Adverse events}

Although it is expected that the intervention will be safe, adverse events (AE) will be pre-specified and reported to the Data Safety and Monitoring Board (DSMB; see below) in accordance with NHMRC position statement of monitoring and reporting of clinical trials. A DSMB will be established that includes an independent researcher and two independent clinicians including one with experience in clinical trials and the other with managing diabetic foot infections. As this is not a systemic intervention, there will be no stopping rules based on any haematological or biochemical parameters.

A severe adverse event (SAE) form will be reported promptly to the DSMB if any of the following occurs:

1. Death from any cause

2. Major limb amputation of the same leg as the index ulcer at any stage up to 12 months from enrolment

3. Major infection of the harvest site as defined as the requirement for admission to hospital, surgical debridement or intravenous antibiotics

An AE form will be reported if any of the following occurs:

1. Readmission for any reason related to infection or deterioration of the index ulcer

2. Minor amputation unrelated to the index ulcer, but on the same foot as the index ulcer (after enrolment)

3. Minor infection of the harvest site as defined by erythema and the requirement for oral antibiotic therapy for this

4. Delayed healing of the harvest site as defined by persistent need for a dressing on the harvest site at or beyond the 4 week visit

\section{Statistical considerations}

\section{Primary and secondary outcomes}

All analyses will be conducted according to the intentionto-treat principle. Baseline characteristics will be compared by treatment group. Effects of treatment on the primary study endpoint (complete healing of the index ulcer at 6 months) will be estimated with the use of unadjusted logistic regression with last observation carried forward for those lost to follow-up. All $P$-values will be two-sided and $P$-values less than 0.05 will be considered to indicate statistical significance. Multiple logistic regression will be applied to adjust for prognostic factors such as vascular insufficiency and site of DFU (fore-, mid- or hind foot) which are strongly correlated with the outcome $(r \geq 0.3)$ $[25,26]$. Binary secondary outcomes at 12 months will be analysed similarly. For continuous secondary outcomes, change score analysis that determines treatment effect based on the difference between baseline and post treatment score (basic adjustment) will be undertaken. Multiple linear regression adjusting for i) the baseline value of the outcome variable in the model (model 1), and ii) model 1 +adjustment for prognostic baseline factors (model 2) will be undertaken. Data will be analysed using IBM SPSS Statistics 25.

\section{Subgroup analyses}

Pre-specified sub-group analyses will be performed using the dichotomous primary study endpoint described above. Sub-groups include ulcer site (categorical variable; fore-, mid- or hind foot, plantar/dorsal), WiFI Clinical Stage [27] at baseline presentation (categorical/ordinal variable; clinical stage 1-4), co-existent moderate to severe renal disease (dichotomous variable; creatinine clearance $\leq 30$ $\mathrm{mL} / \mathrm{min}$, age (dichotomous variable; age $\leq 60$ years) long term diabetic control at presentation (dichotomous variable; $\mathrm{HbA} 1 \mathrm{C} \leq 9 \%$ ), primary surgical procedure performed (dichotomous variable; minor amputation vs. local sharp or surgical debridement).

\section{Interim analysis}

Due to the long-time delay until the primary outcome can be ascertained for each patient, we plan a single interim analysis after the first 80 patients. We estimate that 78 (with continuity correction) patients are required to have $80 \%$ chance of detecting, as significant at the $1 \%$ level, an increase in the primary outcome measure from $45 \%$ in the control group to $85 \%$ in the spray-on skin group [19]. If this threshold is met, the trial will be ceased early. If this threshold is not met, the trial will be completed as described above.

\section{Health economic analyses}

The main perspective of the analyses will be societal. Direct health care costs including spray on skin and usual care treatment costs, hospital inpatient and outpatient costs, including hospital in the home, out-ofhospital medical services and consumables will be estimated. An incremental cost-effectiveness analysis will be performed in which the net costs and net effectiveness of spray on skin will be compared with those of usual 
care and expressed as ratios. All analyses and comparisons will be performed on an intention-to-treat basis. Since the time horizon for the primary endpoint (complete healing of the index ulcer) is 6 months, discounting will not be performed. The confidence intervals for the incremental cost-effectiveness ratio will be estimated using the bootstrap approach with 1000 repeated random samples drawn with replacement from the original data. Bootstrap confidence intervals will be constructed with the bias-corrected percentile method. Data will be analysed using IBM SPSS Statistics 25 [IBM Corporation].

Overall costs will be determined by length of hospital stay, number and nature of operating theatre visits, use of pathology and radiology services, length of "hospital in the home" treatment, direct antibiotic costs, consumables associated with wound management (dressings, debridement, human skin replacements, negative pressure wound therapy dressings; ambulatory nursing attendance, orthotic appliances (prostheses, casting, shoes, insoles). To ascertain data on direct costs for outpatient home care nursing we will extract data from the ambulatory nursing services electronic data collection tool (COMCARE $^{\mathrm{mm}}$ [Docobo]). Outpatient visit costs will be derived from Medicare rebates (for multidisciplinary team visits and single specialty attendances at podiatry, infectious diseases or vascular clinics), whilst drug costs will be estimated from listed drug costs from Pharmaceutical Benefits Scheme (PBS) published listing, or in the case of moxifloxacin, piperacillin-tazobactam, ertapenem and other non-PBS drugs, from direct pharmacy costs. All costs will be adjusted on a year-by-year basis according to the medical services component of the Consumer Price Index to a single financial year for comparisons (2018).

\section{Data management procedures}

Study data will be collected and managed using REDCap [9.2.5 Vanderbilt University] electronic data capture tools hosted at the University of Western Australia [28].

\section{Discussion}

There are substantial barriers to undertaking randomised controlled trials in patients with DFU. Significant heterogeneity in lower limb vascular supply, ulcer location and size, long-term diabetes control, infection extent, antibiotic efficacy, adherence to offloading and psychosocial issues are all factors that affect healing and may compromise the validity of testing a novel intervention to improve cure rates in this group. Restricting entry criteria may reduce heterogeneity in the trial participants, but places limits on generalisability of the results to other patients outside this group. For example, excluding patients with established osteomyelitis in the 'SIDESTEP' trial comparing ertapenem with piperacillin-tazobactam for diabetic foot disease limited generalisability to patients with this common complication [29].

In the case of the spray on skin intervention for the present trial, the goal was to maximise generalisability to as many patients with a DFU at 'moderate' risk of delayed healing. Because the additional costs are likely to be sensitive to the cost of $\mathrm{ReCell}^{\circ}$, smaller wounds with a high likelihood of healing, were not a suitable application for this intervention. Such wounds would include a transphalangeal amputation site in a patient with good blood supply. Likewise, limiting the intervention to a salvage therapy for wounds with an extremely high chance of clinical failure would also compromise the chances of demonstrating success.

Finding the balance between patient heterogeneity, prior knowledge about likelihood of success and the subsequent generalisability of the trial results is difficult and may have implications for the appropriate sample size calculations. In this trial, healing rates greater than $45 \%$ in the standard care arm from published data may not necessarily reflect outcomes for this type of ulcer in our tertiary multidisciplinary unit.

That slow recruitment rate is a challenge to this trial is not unexpected. Studies have demonstrated that only a third of well-funded trials manage to maintain planned recruitment schedules [30]. For the present study, the commonest reason for patients with DFU that would be ordinarily be suitable for the trial is accessibility. Western Australia covers a large area, and our catchment covers patients from distances greater than $500 \mathrm{~km}$. Our experience thus far is that patients $>60$ min travel away may struggle to access ambulatory care services and together with regular travel to FSH precludes recruitment. Our approach to improve patient recruitment was to expand to include RPH as a second site. By replicating the trial infrastructure in another Perth metropolitan tertiary hospital, we hope to improve the catchment of patients. Refusal of eligible patients to participate in this trial has been reassuringly uncommon.

\section{Abbreviations}

AE: adverse event; CT: computer tomography; DFU: diabetic foot ulcer; EQ5D-5 L: European QoL - 5 dimensions survey; FSH: Fiona Stanley Hospital; HbA1c: glycated haemoglobin; HREC: health research ethics committee; LEA: lower extremity amputation; LoS: length of stay; MRI: magnetic resonance imaging; PBS: pharmaceutical benefits scheme; QoL: Quality of life; REDCap: Research Electronic Data Capture; RPH: Royal Perth Hospital; SAE: severe adverse event; SMHS: South Metropolitan Health Service; SSG: split skin graft; T2DM: Type 2 diabetes mellitus; WA: Western Australia

\section{Acknowledgements}

Not applicable.

\section{Authors' contributions}

LM was a major contributor in writing this manuscript. IF, JH \& ER edited the manuscript for publication. LM, PG, IF, MB, FW, PN, JR, KC, EH, ER contributed 
directly to study design procedures, protocol development and implementation of the trial. WD contributed to power calculations and statistical and health economic considerations for this study. All authors read and approved the final manuscript.

\section{Availability of data and materials}

Not applicable. We have not yet generated any data as part of this trial.

\section{Ethics approval and consent to participate}

This trial has been approved by the South Metropolitan Health Service (SMHS) Health Research Ethics Committee (HREC), approval number RGS000000722.

Written informed consent may be obtained at any time prior to randomisation and the commencement of the study.

The participants may withdraw consent at any time throughout the course of the study.

At any time of the study, the principal investigators will be available to answer questions regarding any aspect of the present study. It will be emphasised and articulated clearly to the participants that they are free to decline to participate and that this will not impact on their current or future treatment in any way. The participants or guardian/nearest relative of a participant may withdraw consent at any time throughout the course of the study. The confidentiality of participant information will be maintained at all times.

Informed consent documents and information sheets have been approved for use by the SMHS HREC.

\section{Consent for publication}

Not applicable.

\section{Competing interests}

The WA Department of Health is the main funder of this trial, through the 2017 Research Translation Project Grants (Round 11).

ReCell ${ }^{\oplus}$ was developed in WA by FW, who is an investigator on the study. To avoid perceived conflict of interest, FW assigned all Intellectual Property for ReCell ${ }^{\oplus}$ to a charitable foundation (Fiona Wood Foundation). All ReCell ${ }^{\oplus}$ kits in WA health are used on a not-for-profit basis. In the case of the present study, the ReCell ${ }^{\circledast}$ kits have been donated by the Fiona Wood Foundation for this study of DFU. The manufacturers (Avita Medical) had no role in the study design.

None of the other investigators report a real or perceived conflict of interest for this study.

\section{Author details}

'Department of Infectious Diseases, Fiona Stanley Hospital, 11 Robin Warren Drive, Murdoch, WA 6150, Australia. ${ }^{2}$ Endocrinology Department, Fiona Stanley Hospital, 11 Robin Warren Drive, Murdoch, WA 6150, Australia. ${ }^{3}$ Medical School, University of Western Australia, Crawley, WA 6009, Australia. ${ }^{4}$ Department of Vascular Surgery, Fremantle Hospital, Alma Street, Fremantle, WA 6160, Australia. ${ }^{5}$ State Burns Unit, Fiona Stanley Hospital, 11 Robin Warren Drive, Murdoch, WA 6150, Australia. ${ }^{6}$ Silver Chain Group and School of Nursing and Midwifery, Curtin University, Bentley, WA 6102, Australia. ${ }^{7}$ Podiatry Department, Fiona Stanley Hospital, 11 Robin Warren Drive, Murdoch, WA 6150, Australia. ${ }^{8}$ Burns Department, Fiona Stanley Hospital, 11 Robin Warren Drive, Murdoch, WA 6150, Australia. ${ }^{9}$ Department of Vascular Surgery, Fiona Stanley Hospital, 11 Robin Warren Drive, Murdoch, WA 6150, Australia.

\section{Received: 22 August 2019 Accepted: 30 October 2019} Published online: 15 November 2019

\section{References}

1. Australian Institute of Health and Welfare. Diabetes: Australian facts 2008. In: AlHW C, editor 2008.

2. Bergin SM, Alford JB, Allard BP, Gurr JM, Holland EL, Horsley MW, et al. A limb lost every 3 hours: can Australia reduce amputations in people with diabetes? Med J Aust. 2012;197(4):197-8.

3. Dillon MP, Fortington LV, Akram M, Erbas B, Kohler F. Geographic variation of the incidence rate of lower limb amputation in Australia from 2007-12. PLoS One. 2017;12(1):e0170705.
4. Dillon MP, Kohler F, Peeva V. Incidence of lower limb amputation in Australian hospitals from 2000 to 2010. Prosthetics Orthot Int. 2014;38(2):122-32.

5. Kurowski JR, Nedkoff L, Schoen DE, Knuiman M, Norman PE, Briffa TG. Temporal trends in initial and recurrent lower extremity amputations in people with and without diabetes in Western Australia from 2000 to 2010 Diabetes Res Clin Pract. 2015;108(2):280-7.

6. Baba M, Davis WA, Norman PE, Davis TM. Temporal changes in the prevalence and associates of diabetes-related lower extremity amputations in patients with type 2 diabetes: the Fremantle diabetes study. Cardiovasc Diabetol. 2015;14:152.

7. Payne CB. Diabetes-related lower-limb amputations in Australia. Med J Aust. 2000;173(7):352-4.

8. Lazzarini PA, Hurn SE, Kuys SS, Kamp MC, Ng V, Thomas C, et al. Direct inpatient burden caused by foot-related conditions: a multisite pointprevalence study. BMJ Open. 2016;6(6):e010811.

9. Huang $Y-Y$, Lin C-W, Yang H-M, Hung S-Y, Chen IW. Survival and associated risk factors in patients with diabetes and amputations caused by infectious foot gangrene. J Foot Ankle Res. 2018;11:1.

10. Jeyaraman K, Berhane T, Hamilton M, Chandra AP, Falhammar H. Mortality in patients with diabetic foot ulcer: a retrospective study of 513 cases from a single Centre in the Northern Territory of Australia. BMC Endocr Disord. 2019;19(1):1.

11. Moulik PK, Mtonga R, Gill GV. Amputation and Mortality in New-Onset Diabetic Foot Ulcers Stratified by Etiology. Diabetes Care. 2003;26(2):491-4.

12. Jupiter DC, Thorud JC, Buckley CJ, Shibuya N. The impact of foot ulceration and amputation on mortality in diabetic patients. I: from ulceration to death, a systematic review. Int Wound J. 2016;13(5):892-903.

13. Cheng Q, Lazzarini PA, Gibb M, Derhy PH, Kinnear EM, Burn E, et al. A costeffectiveness analysis of optimal care for diabetic foot ulcers in Australia. Int Wound J. 2017;14(4):616-28.

14. Norman PE, Schoen DE, Gurr JM, Kolybaba ML. High rates of amputation among indigenous people in Western Australia. Med J Aust. 2010;192(7):421.

15. West M, Chuter V, Munteanu S, Hawke F. Defining the gap: a systematic review of the difference in rates of diabetes-related foot complications in aboriginal and Torres Strait islander Australians and non-indigenous Australians. J Foot Ankle Res. 2017;10:48.

16. Driver VR, Fabbi M, Lavery LA, Gibbons $G$. The costs of diabetic foot: the economic case for the limb salvage team. J Am Podiatr Med Assoc. 2010; 100(5):335-41.

17. Jeffcoate WJ, Chipchase SY, Ince P, Game FL. Assessing the outcome of the management of diabetic foot ulcers using ulcer-related and person-related measures. Diabetes Care. 2006;29(8):1784-7.

18. Ndosi M, Wright-Hughes A, Brown S, Backhouse M, Lipsky BA, Bhogal M, et al. Prognosis of the infected diabetic foot ulcer: a 12-month prospective observational study. Diabet Med. 2018;35(1):78-88.

19. De Angelis B, Migner A, Lucarini L, Agovino A, Cervelli V. The use of a non cultured autologous cell suspension to repair chronic ulcers. Int Wound $J$. 2015;12(1):32-9.

20. Chant H, Woodrow T, Manley J. Autologous skin cells: a new technique for skin regeneration in diabetic and vascular ulcers. J Wound Care. 2013;22(10 Suppl):S10-5.

21. Smith-Strom H, Iversen MM, Igland J, Ostbye T, Graue M, Skeie S, et al. Severity and duration of diabetic foot ulcer (DFU) before seeking care as predictors of healing time: a retrospective cohort study. PLoS One. 2017; 12(5):e0177176.

22. Herdman M, Gudex C, Lloyd A, Janssen M, Kind P, Parkin D, et al. Development and preliminary testing of the new five-level version of EQ-5D (EQ-5D-5L). Qual Life Res. 2011:20(10):1727-36.

23. Van Netten JJ, Bus SA, Apelqvist J, Lipsky BA, Hinchliffe RJ, Game FL, et al. Definitions and criteria for diabetic foot disease. IWGDF Guidelines. 2019.

24. Sothornwit J, Srisawasdi G, Suwannakin A, Sriwijitkamol A. Decreased health-related quality of life in patients with diabetic foot problems. Diabetes Metab Syndr Obes. 2018;11:35-43.

25. Pickwell KM, Siersma VD, Kars M, Holstein PE, Schaper NC. Eurodiale c. diabetic foot disease: impact of ulcer location on ulcer healing. Diabetes Metab Res Rev. 2013;29(5):377-83.

26. Lu SH, McLaren A-M. Wound healing outcomes in a diabetic foot ulcer outpatient clinic at an acute care hospital: a retrospective study. J Wound Care. 2017;26(Sup10):S4-S11. 
27. Mills JL, Sr., Conte MS, Armstrong DG, Pomposelli FB, Schanzer A, Sidawy AN, et al. The Society for Vascular Surgery Lower Extremity Threatened Limb Classification System: risk stratification based on wound, ischemia, and foot infection (WIfl). J Vasc Surg. 2014;59(1):220-234 e1-2.

28. Harris PA, Taylor R, Thielke R, Payne J, Gonzalez N, Conde JG. Research electronic data capture (REDCap)--a metadata-driven methodology and workflow process for providing translational research informatics support. J Biomed Inform. 2009;42(2):377-81.

29. Lipsky BA, Armstrong DG, Citron DM, Tice AD, Morgenstern DE, Abramson MA. Ertapenem versus piperacillin/tazobactam for diabetic foot infections (SIDESTEP): prospective, randomised, controlled, double-blinded, multicentre trial. Lancet. 2005;366(9498):1695-703.

30. Reynolds T. Clinical trials: can technology solve the problem of low recruitment? BMJ. 2011;342:d3662.

\section{Publisher's Note}

Springer Nature remains neutral with regard to jurisdictional claims in published maps and institutional affiliations.

Ready to submit your research? Choose BMC and benefit from:

- fast, convenient online submission

- thorough peer review by experienced researchers in your field

- rapid publication on acceptance

- support for research data, including large and complex data types

- gold Open Access which fosters wider collaboration and increased citations

- maximum visibility for your research: over $100 \mathrm{M}$ website views per year

At $\mathrm{BMC}$, research is always in progress.

Learn more biomedcentral.com/submissions 